\title{
Solvent Effects on the Racemization of Optically Active Biphenyl Derivatives
}

\author{
LARS BALTZER and NILS-ÅKE BERGMAN
}

Department of Organic Chemistry, University of Göteborg and Chalmers University of Technology, S-412 96 Göteborg, Sweden

The biphenyl derivative 2,2'-dihydroxy-6,6'dinitrobiphenyl has been synthesized. As a minor by-product 2-hydroxy-2'-methoxy-6,6'-dinitrobiphenyl was also obtained. The inversion rates of the former and its dianion and of the anion of the latter have been measured in a range of solvents.

A large rate increase is observed for the dianion of $2,2^{\prime}$-dihydroxy-6,6'-dinitrobiphenyl when the solvent is changed from water to $95 \mathrm{~mol} \%$ of dimethyl sulfoxide in water. The effect is interpreted as being due to desolvation of the dianion by dimethyl sulfoxide.

The kinetic solvent isotope effect on the inversion of the dianion has been measured. In water the effect is negligible, and added dimethyl sulfoxide has no effect on the isotopic rate ratio. In methanol $k^{\mathrm{OH}} / k^{\mathrm{OD}}$ $=0.93 \pm 0.02$. The effect is somewhat more pronounced in $25 \mathrm{~mol} \%$ of dimethyl sulfoxide in methanol, $k^{\mathrm{OH}} / k^{\mathrm{OD}}=0.83$. The increase in the strength of the effect is probably due to increased fractionation because of desolvation.

The biphenyl frame-work has provided a very useful model system for a large variety of investigations. A multiplicity of derivatives has been used for the study of steric, ${ }^{1}$ salt $^{2}$ and solvent effects. ${ }^{3}$ A particularly beautiful example is the elucidation of the nature of steric isotope effects with protium and deuterium. ${ }^{4}$ In view of the very simple and wellknown geometry of the biphenyl inversion reaction we decided to use it for our study of hydrogen-bond types of interaction between oxyanions and hydroxylic solvents. In an earlier investigation, ${ }^{5}$ the rate of racemization of the dianion of 6-nitrodifenic acid was determined in light and heavy water. The results were interpreted in terms of the fractionation theory ${ }^{6}$ and a model was suggested where the

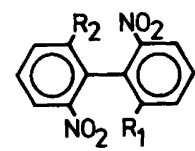

I $R_{1}=R_{2}=0^{-}$

II $\mathrm{R}_{1}=\mathrm{R}_{2}=\mathrm{OH}$

II $R_{1}=O, R_{2}=O H_{3}$

v $\mathrm{R}_{1}=\mathrm{OH}, \mathrm{R}_{2}=\mathrm{OCH}_{3}$

Scheme 1.

Y $R_{1}=R_{2}=O \mathrm{H}_{3}$

numbers of solvating molecules in the initial and transition states were given.

In order to extend the study, an optically active biphenyl derivative, 2,2'-dihydroxy-6,6'-dinitrobiphenyl (II), with the possibility of more concentrated charges in the ortho positions, has been synthesized and the range of solvents has been extended. A study of this kind provides some insight into the phenomena of solvation in sterically hindered systems, and the results obtained should be useful in discussing other sterically hindered systems where our knowledge of the solvation is very limited, e.g., the transition states of chemical reactions.

\section{EXPERIMENTAL}

2,2'-Dimethoxy-6,6'-dinitrobiphenyl (V) was prepared according to the method of Adams and Finger ${ }^{7}$ with the following modifications. Methylation of 2-chloro-3-nitrophenol was carried out according to Brändström. ${ }^{8}$ Ullman coupling of 2-chloro-3-nitroanisole was performed in a melt, according to standard procedures. ${ }^{9}$ The compound V so obtained, consisted of yellow crystals, m.p. 228 $-230^{\circ} \mathrm{C}$ (lit. ${ }^{7} 226-228^{\circ} \mathrm{C}$ ).

2,2'-Dihydroxy-6,6'-dinitrobiphenyl (II) was obtained from $\mathrm{V}$ by cleavage of the alkyl aryl ether using $\mathrm{AlCl}_{3}$ in the dry. ${ }^{10}$ The compound $\mathrm{V}(1.8 \mathrm{~g})$ was carefully ground in a mortar with $4 \mathrm{~g}$ of $\mathrm{AlCl}_{3}$, 
under nitrogen. The material was put in a roundbottomed flask equipped with a drying tube, and the flask was heated at $90^{\circ} \mathrm{C}$ overnight. The reactants were again ground in the mortar with an additional portion of $\mathrm{AlCl}_{3}, 1 \mathrm{~g}$, and heated at $110^{\circ} \mathrm{C}$ for two hours. The contents of the flask were poured onto ice. The mixture was made basic and filtered, the deeply red filtrate acidified and filtered to give yellow crystals. Esterification with acetyl chloride, chromatography on silica gel with dichloromethane followed by hydrolysis of the two products in $0.5 \mathrm{M}$ $\mathrm{NaOH}$ in $50 \%$ ethanol-water (v/v) gave the following yields: the compound II, $180 \mathrm{mg}(11 \%)$ m.p. $295^{\circ} \mathrm{C}$ and IV, $30 \mathrm{mg}$ m.p. $223^{\circ} \mathrm{C}$. Some unreacted starting material $(0.6 \mathrm{~g})$ could also be recovered. An attempt to cleave the ether groups by $\mathrm{BBr}_{3}{ }^{11}$ was unsuccessful.

Resolution of $I I$ and $I V$. The crude product after cleavage with $\mathrm{AlCl}_{3}$ was considered pure enough for this step. In order to resolve the compounds they were converted into diastereomeric esters ${ }^{12}$ with the acid chloride of $(+)-2$-phenylbutyric acid (Norse Laboratories, Inc.). The acid chloride was prepared by treatment of the acid with excess of freshly distilled $\mathrm{SOCl}_{2},{ }^{13}$ and the optical activity of the obtained acid chloride was $[\alpha]_{\mathrm{D}}^{25}=+94.5$ (c. 2.25 , benzene). The mixture of diastereomeric esters was separated by HPLC using a stainless steel column $(30 \mathrm{~cm}, 7.7 \mathrm{~mm}$ I.D.) packed with silica gel (Porsil, Waters Ass.) and cyclohexane - acetone 9:1 (v/v) as eluent. Base-line separation was nearly achieved but due to low solubility in suitable solvents no attempt was made to estimate optical purity. The optically active esters were hydrolyzed as described above. Melting point of (+)- II, $267^{\circ} \mathrm{C}$ (decomposes). Optical activity of $(+)$ - II: $[\alpha]_{D}^{20}=+675(c .=0.114$, methanol).

The identity of II was established from mass spectrometric analysis and by NMR. The identity of IV was established from NMR. Both compounds were those expected from the synthetic procedure.

Solvents from the following manufacturers were used without further purification. Methanol (May \& Baker, Methanol Anhydrous, water less than $0.01 \%$, w/w), methanol-O-d (Ciba-Geigy 99.7 atom \% D) and in later runs 99.9 atom \% D, ultrapur (when the first-mentioned quality was withdrawn from the market), DMSO (Riedel-de Haën, getrocknet für Analyse, less than $0.01 \%$ water, w/w), DMSO- $d_{6}$ (Ciba-Geigy 99.5 atom \% D) and deuterium oxide (Ciba-Geigy 99.7 atom \% D). Light water was deionized, distilled and boiled out under nitrogen. Ethanol (Kemetyl AB, absolute ethanol, 99.5\%) and $t$-butanol (FLUKA, tert-butyl alcohol, puriss. p.a.) were purified according to the method of Bjerrum and Lund. ${ }^{14}$ To test the water content of some of the solvents, the commercial methanol and the purified ethanol and $t$-butanol were subjected to Karl
Fischer titration. In no case did the water content exceed $0.012 \%(\mathrm{w} / \mathrm{w})$.

Base solutions were prepared in the following way. Sodium hydroxide from commercially available ampoules, sodium deuteroxide by dilution of a commercially available solution, (Merck, Natronlauge- $d_{1}$ ) with deuterium oxide. Sodium methoxide and ethoxide and potassium $t$-butoxide by dissolving clean sodium or potassium metal in the respective solvent. Preparation of base solutions was carried out under nitrogen. All base solutions were titrated against $0.1 \mathrm{M} \mathrm{HCl}$ or potassium hydrogen phthalate. Mixed solvents were prepared by weight and base solutions were diluted by weight.

The kinetic runs were performed in three different ways depending on the reaction rate and on the optical density of the solutions. The reactions in water and a few of the reactions in DMSO - water were followed in a polarimeter cell of a Zeiss Digital Polarimeter Old 5, that was connected to an automatic data collecting assembly. When the solutions were too coloured to allow the polarimeter to read accurately the optical rotation of the reaction solution, sealed ampoules had to be used during the reaction, and when the reactions had half-lives of $20 \mathrm{~min}$ or less the reaction solution was kept in a gas-tight bottle sealed with a septum and the samples were collected with a Hamilton syringe. In the ampoule and gas-tight bottle experiments the aliquots were acidified and the optical rotation of the undissociated compound was determined.

Treatment of data. The rate constants were calculated with a least-squares program or with the program PROGAEXP that fits an exponential expression to the experimental data. This program was used in the polarimetric runs.

Check experiments. On some occasions racemizations were run for more than ten half-lives in order to check the kinetics. No deviations from firstorder kinetics could be detected. Several of the kinetic runs were followed by UV/VIS spectroscopy to detect any deterioration of the substrate. Only in one solvent, ethanol, did this occur. These reactions were therefore not monitored for more than one half-life, and the results from them should be treated with some caution.

The maximum of the longest wave-length absorption of I appears at $395 \pm 5 \mathrm{~nm}$ in water, methanol, ethanol and $t$-butanol. It is sensitive to the DMSO content in the binary mixtures, and it is redshifted as much as $50 \mathrm{~nm}$ at high DMSO concentrations. In $t$-butanol solutions of predominant DMSO content a new absorption band is observed at $495 \pm 5 \mathrm{~nm}$. Its intensity grows with $\mathrm{x}_{\mathrm{DMso}}$. The band is also seen in $t$-butanol in the presence of excess of crown-ether. The significance of the new absorption is not known. 
Table 1. Racemization of $I$ in different solvent systems. Counterion: $\mathrm{Na}^{+}\left(\mathrm{K}^{+}\right.$in $t-\mathrm{BuOH}$ and mixtures of it). Temperature $333.15 \mathrm{~K}$.

\begin{tabular}{|c|c|c|}
\hline Solvent & $\begin{array}{l}\text { Mol fraction } \\
\text { DMSO }\end{array}$ & $k \times 10^{-5} / \mathrm{s}^{-1 a}$ \\
\hline $\mathrm{H}_{2} \mathrm{O}-\mathrm{DMSO}$ & $\begin{array}{l}0 \\
0 \\
0 \\
0.251 \\
0.500 \\
0.516 \\
0.751 \\
0.954\end{array}$ & $\begin{array}{l}0.172 \pm 0.003 \\
2.26 \pm 0.02^{b} \\
2.31 \pm 0.02^{b, c} \\
1.31 \pm 0.06 \\
7.49 \\
7.72 \\
24.9 \\
100\end{array}$ \\
\hline $\mathrm{D}_{2} \mathrm{O}-\mathrm{DMSO}$ & $\begin{array}{l}0 \\
0 \\
0 \\
0.249 \\
0.251 \\
0.503 \\
0.752 \\
0.951\end{array}$ & $\begin{array}{l}0.165 \pm 0.009 \\
2.20 \pm 0.02^{b} \\
2.24 \pm 0.02^{b, c} \\
1.30 \\
1.29 \\
7.06 \\
25.7 \\
89.1\end{array}$ \\
\hline MeOH - DMSO & $\begin{array}{l}0 \\
0.246 \\
0.251 \\
0.253 \\
0.259 \\
0.265 \\
0.606 \\
0.820 \\
0.947 \\
0.950\end{array}$ & $\begin{array}{c}0.927 \pm 0.002 \\
3.20 \\
3.27 \\
3.21 \\
3.43 \\
3.80 \\
15.6 \\
32.2 \\
57.7 \\
74.9\end{array}$ \\
\hline MeOD-DMSO & $\begin{array}{l}0 \\
0.247 \\
0.264 \\
0.591 \\
0.793\end{array}$ & $\begin{array}{l}0.992 \pm 0.009 \\
3.90 \\
4.15 \\
15.0 \\
27.6\end{array}$ \\
\hline$t$-BuOH -DMSO & $\begin{array}{l}0 \\
0 \\
0.131 \\
0.263 \\
0.507 \\
0.761\end{array}$ & $\begin{array}{c}8.40 \pm 0.07 \\
145 \pm 6^{d} \\
7.52 \\
7.77 \\
20.9 \\
78.3\end{array}$ \\
\hline EtOH & 0 & 1.17 \\
\hline
\end{tabular}

${ }^{a}$ The values are mean values of at least two runs and error limits correspond to maximum deviation from the mean value. Single measurements are given without error limits. In most runs the error limits within one run are less than $2 \%$, but at high DMSO concentrations the reproducibility probably is not better than $\pm 5 \%$ because of the dramatic effect of fortuitous water. ${ }^{b}$ Temperature 353.15 K. ${ }^{c}$ In the excess of cryptand 2.2.1. ${ }^{d}$ In the presence of 18-crown-6.
In order to investigate the influence of the ionic strength on the rate of the reaction of $I$ in water, some runs were performed with varying concentration of $\mathrm{NaOH}(0.05-0.15 \mathrm{M})$. No significant variation of the rate could be detected within the studied concentration interval.

\section{RESULTS}

The rate of racemization of $I$ has been determined in a number of solvents and the results are given in Table 1 . The rate increases by a factor of 600 , relative to that in water, when the reaction is run in $95 \mathrm{~mol} \%$ of dimethyl sulfoxide (DMSO) in water. Comparing methanol, ethanol and tertiary butanol the rate increases in a way that parallels their respective basicities. The importance of ion-pairing to the rate of racemization has also been investigated and in all solvents but those including butanol, it has been found to be negligible. The conclusion was drawn from kinetic runs (water) in the presence of cation complexing agents, the rates being indistinguishable from those obtained in the absence of such agents, and from electronic spectra.

In methanol and ethanol, the spectra were identical before and after addition of a crown ether or a cryptand. In $t$-butanol a batochromic shift is observed on addition of crown ether.

The effect on the rate of reaction by substitution of deuterium for protium in the hydroxylic positions of the solvent molecules has been determined in water, methanol and their mixtures with DMSO, Table 2. The effect in water is very weak, and addition of DMSO does not have any significant influence on the isotopic rate ratio, the apparent difference at high mol fractions of DMSO (Table 1) being probably due to the large uncertainty in the determinations. In methanol, however, the isotope

Table 2. Kinetic solvent isotope effect on the racemization of $I$ in various solvents. Temperature $333.15 \mathrm{~K}$.

\begin{tabular}{lll}
\hline Solvent & $\begin{array}{l}\text { Mol fraction } \\
\text { DMSO }\end{array}$ & $k^{\mathrm{OH}} / k^{\mathrm{OD}}$ \\
\hline $\mathrm{L}_{2} \mathrm{O}$ & 0 & $1.042 \pm 0.07$ \\
$\mathrm{~L}_{2} \mathrm{O}$ & 0 & $1.027 \pm 0.018^{a}$ \\
$\mathrm{MeOH}-\mathrm{DMSO}$ & 0 & $0.93 \pm 0.02$ \\
& 0.25 & 0.83 \\
& 0.60 & $\sim 1$ \\
\hline
\end{tabular}

${ }^{a}$ Temperature $353.15 \mathrm{~K}$. 
Table 3. Racemization of II and III. Counterion $\mathrm{Na}^{+}$ with III. Solvent $\mathrm{H}_{2} \mathrm{O}-\mathrm{DMSO}$. Temperature $353.15 \mathrm{~K}$.

\begin{tabular}{lll}
\hline Substrate & $\begin{array}{l}\text { Mol fraction } \\
\text { of DMSO }\end{array}$ & $k \times 10^{-5} / \mathrm{s}^{-1 a}$ \\
\hline II & 0 & 0.028 \\
& 0.99 & 0.0893 \\
III & 0 & $0.871 \pm 0.022$ \\
& 0.503 & 20.0 \\
\hline
\end{tabular}

${ }^{a}$ See footnote ${ }^{a}$ in Table 1.

effect is pronounced, giving $k^{\mathrm{OH}} / k^{\mathrm{OD}}=0.93 \pm 0.02$, and in $25 \mathrm{~mol} \%$ of DMSO in methanol the rate constant ratio is decreased even further.

For comparison with I, the rate of racemization of 2,2'-dihydroxy-6,6'-dinitrobiphenyl (II) and 2hydroxy-2'-methoxy-6,6'-biphenyl anion (III) have been studied in some solvents and the results are given in Table 3. The biphenol, II, is slower than I by a factor of 80 at $353 \mathrm{~K}$ in water, and in mixtures of DMSO and water of mol fractions of DMSO larger than 0.95 , the reaction of $I$ is more than 1100 times faster. The reaction rate of III is slightly slower than that of $I$ in water at $353 \mathrm{~K}$, and the rate in an equimolar mixture of DMSO and water is increased by a factor of 23 relative to the former solvent. For comparison, the corresponding increase for I at 353 $\mathrm{K}$ amounts to a factor of 44 .

\section{DISCUSSION}

The solvent effect on the rate of racemization of biphenyl derivatives is generally very small. In one investigation the rates in widely different solvents were all within a factor of five. ${ }^{3}$ The large increase presented here is therefore indicative of specific solute-solvent interactions. In the following, solvent effects and solvent isotope effects on the rate of racemization, together with spectroscopic data, will be used to assign numbers of hydrogen-bonded solvating molecules to the reactant and transition states.

Solvent effects. The role of DMSO in mixtures with hydroxylic solvents is to act as an acceptor of hydrogen bonds from its cosolvent and to desolvate the anions. The rate increase in the racemization of I is consequently interpreted in these terms.

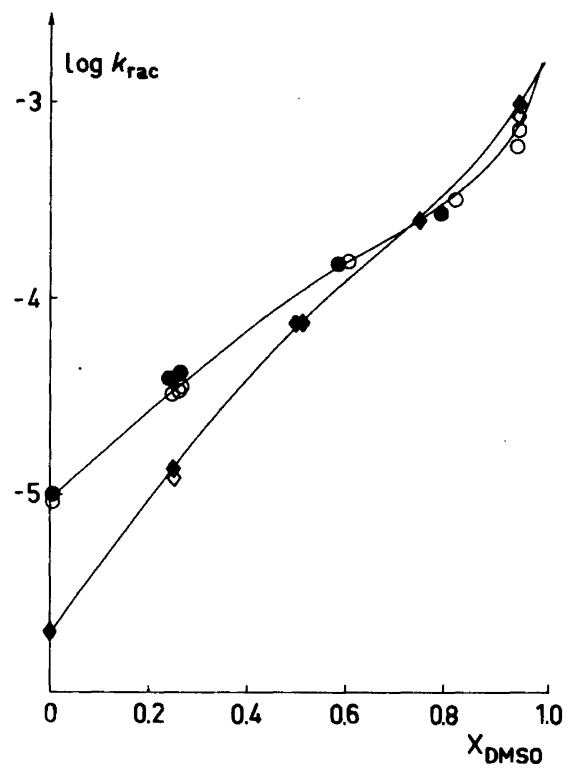

Fig. 1. Variation of the logarithm of the rate constant for racemization of $\mathrm{I}$ in mixtures of hydroxylic solvents with DMSO. Temperature $333.15 \mathrm{~K}$. MeOD/DMSO; O, MeOH/DMSO; $\mathrm{H}_{2} \mathrm{O} / \mathrm{DMSO} ; \diamond, \mathrm{D}_{2} \mathrm{O} / \mathrm{DMSO}$.

The charged oxygen atoms of I are solvated by a number of hydroxylic molecules. In the inversion reaction, the ortho substituents have to pass each other and a sterically hindered transition state is reached in the process. Some of the solvating molecules are then scraped off. In the pure hydroxylic solvent the reactant carries more solvent molecules than the transition state and is more destabilized on addition of DMSO to the solution. The result is that when the DMSO content is increased in the solution, the rate of the reaction is enhanced (Fig. 1). If this interpretation is correct a similar effect would also be expected in hydrogen bonding solvents where the hydrogen-bond donating ability is weaker than in water. The results from kinetic runs in some alcohols, Table 1, are in agreement with this prediction.

Comparison with II and III that have fewer hydrogen-bond accepting sites than I also corroborates our hypotesis. The racemization rate of II is not enhanced much, only a factor of three on transfer from water to $99 \mathrm{~mol} \%$ DMSO - water (Table 3), an increase that is of the same magnitude as those already reported for substrates without specific interactions with the solvent. The anion III 
has only one charged oxygen atom and should be solvated by about half as many molecules as I. If that is so, the number of solvent molecules that will have to be scraped off is also expected to be half the number for I. A rate increase for III is therefore anticipated that is weaker than that of $\mathrm{I}$, corresponding to a decrease in the free-energy of activation by half the amount for $\mathrm{I}$. This is qualitatively born out, Table 3 .

However, one must realize at this point that the making or breaking of hydrogen bonds should contribute to the enthalpy of activation, a quantity that is unknown to us. Without any knowledge of the activation parameters the question might be raised whether we are, in fact, dealing with an entropy effect. An increase in the activation entropy of $59 \mathrm{~J} / \mathrm{mol} \mathrm{K}$ would account for the variation in rate between water and DMSO (Fig. 1) observed for compound I. This is not very likely to be the explanation as entropies of activation in the biphenyl system are generally negative and small, -21 to $-63 \mathrm{~J} / \mathrm{mol} \mathrm{K}$. Thus, it seems unlikely that the solvent effects on the rate should be mainly entropy effects, but a minor contribution may be included.

Another alternative explanation might be that there is some strong interaction between DMSO and the transition state of $I$ that accelerates the racemization. The rationalization for such an argument would be found in the fact that large polarizable anions show solvent activity coefficients of less than unity in dipolar solvents, when hydroxylic ones are used as reference solvents. ${ }^{15}$

Experimental evidence which makes this explanation rather unlikely comes from a comparison of the rate of racemization of $I$ in $t$ butanol and DMSO, respectively, (for possible pitfalls with respect to the $t$-butanol results, see above). In a plot of $\log k_{\mathrm{rac}}$ versus the mol fraction of DMSO, $x_{\text {DMSO }}$, Fig. 1 , an extrapolation to $x_{\text {DMSO }}=1$ gives a good measure of the rate in pure DMSO. The rate in DMSO coincides very closely with that in $t$ butanol in the presence of excess crown ether. Unless it is a pure coincidence, the only explanation appears to be that $t$-butanol is a very weak hydrogen-bonding solvent and that $I$ is practically unsolvated in both solvents. In that case, the conclusion is inescapable that polarization of the transition state contributes negligibly to the reaction rate, $t$-butanol and DMSO being too different as solvents, with respect to most physical properties, ${ }^{16}$ to give similar rates otherwise.
Salt effects. Apart from a non-specific "medium" effect, here of negligible magnitude (see Experimental), chelate formation with a cation may occur when negatively charged groups are situated in the ortho positions of the two rings. A slower reaction is expected with the chelate than with the free dianion. The rate of racemization of $\mathrm{I}$ in $t$ butanol with the potassium ion as counter-ion is increased 17 times when 18-crown-6 is added in excess, (Table 1). We take this as proof of a tendency for chelate formation between I and the potassium ion.

Provided that the inversion rate of the chelate is negligible the observed rate constant in such a case is $^{2 a}$

$k_{\mathrm{obs}}=k_{\mathrm{rac}} \frac{1}{1+K\left[\mathrm{M}^{+}\right]}$

where $\left[\mathrm{M}^{+}\right]$is the concentration of cations, $K$ is the equilibrium constant for chelate formation,

$$
K=\frac{\left[\mathrm{IM}^{+}\right]}{[\mathrm{I}]\left[\mathrm{M}^{+}\right]}
$$

In the presence of excess of a powerful complexing agent, no chelation with I occurs, $k_{\mathrm{obs}}=k_{\mathrm{rac}}$, and comparison with the observed rate constant in the absence of complexing agent, allows calculation of $K$. In the present case it has been determined as $K$ $=230 \pm 30 \mathrm{M}^{-1}$.

In the absence of a complexing agent the rate is enhanced when the mol fraction of DMSO in $t$ butanol is increased above 0.25 and approaches that in pure DMSO, as estimated from the methanol and water mixtures with DMSO, (Table 1).

In water, the addition of cryptand $(2,2,1)$ does not significantly change the rate of reaction. The electronic spectra of $I$ in methanol and ethanol are not affected by the addition of crown ether. We conclude that chelate formation is of negligible importance in these solvents, but would like to add that here the sodium ion has been the counter-ion.

Solvent isotope effects. To elucidate the possible use of fractionation theory to assign numbers of molecules to solvating positions, the kinetic solvent isotope effect on the rate of racemization of I has been determined in water, methanol and their mixtures with DMSO, Table 2. Adapted to the present conditions, the theoretical expression describing the rate-constant ratio for the 
racemization reaction in isotopic solvents is given by (1). ${ }^{5}$

$k^{\mathrm{OH}} / k^{\mathrm{OD}}=\prod_{i} \phi_{\mathrm{R} i} / \prod_{j} \phi_{\mathrm{Tj}}$

The $k$ 's are the rate constants for racemization in deuterated $(O D)$ or protiated $(O H)$ solvent. The fractionation factors $\phi$ are given for each fractionating position in the reactant $(R)$ and the transition state $(\mathrm{T})$. The interpretation may be expected to be complicated since all parameters are expected to change on going into DMSO mixtures. The magnitude of $\phi$ can, for the present purpose, be given the simplified meaning that it is lower the stronger the hydrogen bond is.

The solvent isotope effect in water is very weak, a surprising fact at first, but probably due to cancellation between the denominator and the numerator of eqn. 1 . Addition of DMSO does not alter the magnitude of the isotope effect and, as mentioned above, the apparent effect at high mol fractions of DMSO merely reflects the experimental difficulties at those solvent compositions.

In methanol the effect is clearly perceivable and in $25 \mathrm{~mol} \%$ of DMSO in methanol the rate constant ratio decreases even more, but at $60 \mathrm{~mol} \%$ of DMSO the effect is gone (Table 2). In methanol there is undoubtedly a difference in fractionation in the solvation shell of the reactant and the transition state. The effect is not impressive, however, so it is quite possible that some of the solvating molecules are still present when the transition state is reached, giving rise to some cancellation and diminishing the effect. The reason why the solvent isotope effect is strengthened when a dipolar solvent is added is that the contribution to the kinetic solvent isotope effect (1) from the reactant, compared to the transition state, is increased because of desolvation.

Such behaviour has been noted before ${ }^{17}$ and is not restricted to desolvation by a dipolar nonhydroxylic solvent. The same effect is achieved by a steric obstacle that keeps the solvent away from the hydrogen-bonding sites, as can be deduced from the run in water where the isotope effect is nearly invisible in spite of the fact that it is very likely that there should be appreciable fractionation in the solvation shell of I. The evidence for this comes from an NMR experiment where an isotope effect on the chemical shift of the hydroxylic hydrogens has been established on addition of $0.61 \mathrm{M}$ of $\mathrm{I}^{18}{ }^{18}$ There are two reasons for an isotope effect to occur on the chemical shift of hydrogen. One is an anharmonicity effect ${ }^{19}$ that occurs in systems with strong hydrogen bonds. The other reason is isotopic fractionation. ${ }^{20}$ Under the assumption that the potential well does not exhibit anharmonicity we may conclude that fractionation occurs.

Solvation model. From the results in the mixed solvents, some conclusions may be drawn about the detailed solvation of $\mathrm{I}$.

Recollecting that in the transition-state theory ${ }^{21}$ any by-product in the formation of the activated complex appears in the denominator of the expression for the observed rate, we have

Rate $=k_{\mathrm{o}} \times \frac{f_{1}}{f_{\mathrm{S}}^{p} f_{\neq}} \times \frac{[(+)-\mathrm{I}]}{[\mathrm{S}]^{p}}$

In the present case the by-products may be identified with the $p$ hydroxylic solvent molecules, $S$, that are scraped off in the coplanar conformation of I. The symbol $f$ stands for activity coefficient and $k_{0}$ is the rate constant in the standard state. We choose the pure hydroxylic solvent as standard state.

Taking logarithms of the pseudo first-order observed rate constant $\left(k_{\text {obs }}\right),{ }^{22}$ we find

$\log \left(k_{\text {obs }} / k_{\mathrm{o}}\right)=-p \log a_{\mathrm{s}}+\log \left(f_{\mathrm{l}} / f_{\ddagger}\right)$

A plot of $\log \left(k_{\mathrm{obs}} / k_{\mathrm{o}}\right)$ versus $\log a_{\mathrm{s}}$ should give a slope of $-p$. The second term is also a function of the composition of the solvent. In the limit as the composition of the solvent tends toward that of the pure hydroxylic solvent, the slope should be affected very little by the activity-coefficient ratio.

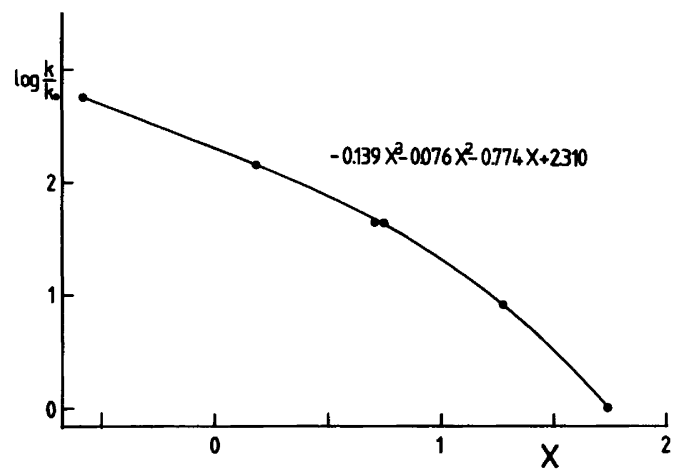

Fig. 2. Plot of $\log k / k_{\mathrm{o}}$ versus $x=\log a_{\mathrm{H}_{2} \mathrm{O}}, k=k_{\mathrm{obs}}$ and $k_{o}$ (rate constant in $\mathrm{H}_{2} \mathrm{O}$ ) are obtained from Table 1 . $a_{\mathrm{H}_{2} \mathrm{O}}$ is calculated from literature data, see text. The solid curve is given by the polynomial. 


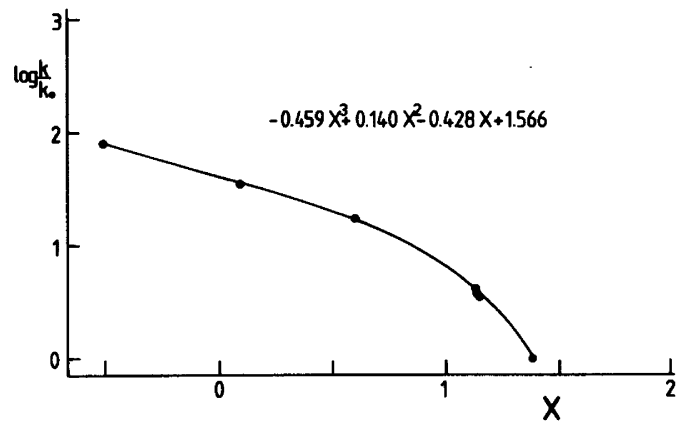

Fig. 3. Plot of $\log k / k_{\mathrm{o}}$ versus $x=\log _{\mathrm{MeOH}} k=k_{\mathrm{obs}}$ and $k$ (rate constant in $\mathrm{MeOH}$ ) are obtained from Table 1. $a_{\mathrm{MeOH}}$ is calculated from literature data, see text. The solid curve is given by the polynomial.

The results for the DMSO - water mixtures are presented in Fig. 2 and those for the DMSO - methanol mixtures are given in Fig. 3. The activity $\left(a_{\mathrm{s}}\right)$, in a mixture of a hydroxylic solvent with DMSO has been defined as $a_{\mathrm{s}}=$ [hydroxylic solvent $] \times f_{\mathrm{s}}, f_{\mathrm{s}}$ being the activity coefficient of the hydroxylic solvent in that mixture. ${ }^{23,24}$ The concentration of the hydroxylic solvent in a given mixture has been calculated from published density data. ${ }^{25,26}$ The experimental data are fitted to polynomials from which the slopes at $x_{\mathrm{DMSO}}=0$ are obtained.

In DMSO-water $p=2.3$ and for DMSO - methanol, $p=2.7$. For simplicity, the solvation numbers are assumed to be integers ( 2 and 3 ).

The upper limit of the solvation number of $I$ is set by the number of available electron pairs on the charged oxygen atoms. The lower limit is zero, but, from the NMR experiment (see above) where an isotope effect on the chemical shift of the hydroxylic hydrogens was obtained, it is obvious that the reactant must carry solvating molecules. From the absence of a kinetic solvent isotope effect in water we conclude that this is true also for the transition state.

A further restriction on the solvation numbers arises from the symmetry of the activated complex where the oxygens are at a maximum distance from each other. The solvation shells are independent of each other and the number of solvating molecules should thus be an even number.

This rather simple picture sums up to a detailed model of the solvation of I. In water the reactant carries 6 or 4 molecules of water and the transition state 4 or 2 , respectively. In methanol the corresponding figures are 5 for the reactant and 2 for the transition state. The underlying assumption that the solvation numbers have to be integers is, of course, an open question.

Acknowledgements. We would like to thank Professor Lars Melander for his interest in the present work and for many fruitful duscussions concerning problems in connection with it. Financial support from the Swedish Natural Science Research Council is gratefully acknowledged.

\section{REFERENCES}

1. Shriner, R. L., Adams, R. and Marvel, C. S. In Gilman, H., Ed., Organic Chemistry An Advanced Treatise, 2nd Ed., Wiley, New York 1958, Chapter 4; Bott, G., Field, L. D. and Sternhall, S. J. Am. Chem. Soc. 102 (1980) 5618.

2. a. Berntsson, P. and Carter, R. E. Acta Chem. Scand. 22 (1968) 2141; b. Berntsson, P., Wanger, M. and Carter, R. E. Acta Chem. Scand. 21 (1967) 879; Graham, W. H. and Leffler, J. E. J. Phys. Chem. 63 (1959) 1274; Leffler, J. E. and Graybill, B. M. J. Phys. Chem. 63 (1959) 1457.

3. Leffler, J. E. and Graham, W. H. J. Phys. Chem. 63 (1959) 687.

4. Carter, R. E. and Melander, L. Adv. Phys. Org. Chem. 10 (1973) 1.

5. Bergman, N.-Å. and Baltzer, L. Acta Chem. Scand. A 31 (1977) 343.

6. Gold, V. Trans. Faraday Soc. 56 (1960) 255; Kresge, A. J. Pure Appl. Chem. 8 (1964) 243. For a recent text on the subject see Melander, L. and Saunders, W. H., Jr. Reaction Rates of Isotopic Molecules, Wiley, New York 1980, Chapter 7.

7. Adams, R. and Finger, G. C. J. Am. Chem. Soc. 61 (1939) 2828.

8. Brändström, A. Preparative Ion Pair Extraction, Swedish Academy of Pharmaceutical Sciences, Stockholm 1976, p. 155.

9. Fanta, P. E. Chem. Rev. 64 (1964) 613.

10. Becker, H.-D. Acta Chem. Scand. 16 (1962) 78.

11. Baumgarten, H. E. Org. Synth. Coll. Vol. 5 (1973) 412.

12. Knauf, A. E., Shildneck, P. R. and Adams, R. J. Am. Chem. Soc. 56 (1934) 2109.

13. Samdahl, B. and Berg, B. Bull. Soc. Chim. Fr. (1949) 461, 463.

14. Fieser, L. F. Experiments in Organic Chemistry, D. C. Heath and Company, Boston 1957, p. 286.

15. Parker, A. J. Chem. Rev. 69 (1969) 1.

16. Reichardt, C. Solvent Effects in Organic Chemistry, Verlag Chem., New York 1979, p. 270.

17. Baltzer, L. and Bergman, N.-Å. J. Chem. Soc. Perkin Trans. 2 To be published. 
18. Baltzer, L. Unpublished results.

19. Altman, L. J., Laungani, D., Gunnarsson, G., Wennerström, H. and Forsén, S. J. Am. Chem. Soc. 100 (1978) 8264.

20. Saunders, M. Personal communication.

21. Glasstone, S., Laidler, K. J. and Eyring, H. The Theory of Rate Processes, MoGraw-Hill, New York and London 1941, p. 184.

22. A similar treatment of acid catalysis in strongly acidic solutions has been used by Bunnett, J.F. J. Am. Chem. Soc. 83 (1961) 4956, 4968, 4973, 4978.

23. Cox, B. G. and McTigue, P. T. Aust. J. Chem. 20 (1967) 1815.

24. Quitzsch, K., Ulbrecht, H. and Geischer, G. Z. Phys. Chem. Leipzig 234 (1967) 33.

25. Tommila, E. Acta Chem. Scand. 20 (1966) 923.

26. Tommila, E. and Savolainen, M. Acta Chem. Scand. 20 (1966) 946.

Received May 5, 1981. 